\title{
Kinesio taping and low-level laser therapy in breast cancer-related lymphedema in an arm with arteriovenous fistula for hemodialysis
}

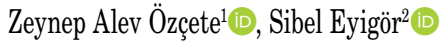 \\ ${ }^{1}$ Department of Physical Medicine and Rehabilitation, Health Sciences University, Izmir Tepecik Training and Research Hospital, Izmir, Turkey \\ ${ }^{2}$ Department of Physical Medicine and Rehabilitation, Ege University Faculty of Medicine Tepecik Training and Research Hospital, Izmir, Turkey \\ Received: July 29, 2018 Accepted: January 04, 2019 Published online: May 18, 2020
}

\begin{abstract}
Secondary lymphedema mainly occurs as a result of impairment or obstruction of the lymphatic system. Although complex decongestive therapy is recognized as the best management technique of lymphedema, we encounter various patient profiles in our clinical practice and may need to apply alternative treatment options. In this article, we report a 57-year-old female patient with breast cancer-related lymphedema and arteriovenous (AV) fistula for hemodialysis in the same arm. We performed low-level laser therapy treatment for 12 sessions, kinesio taping, and remedial exercises to the patient as the compression part of complex decongestive therapy was contraindicated in the AV fistula. Self-manual lymphatic drainage training and skin care education were also given. The arm volume difference was decreased from $691 \mathrm{~mL}$ to $454 \mathrm{~mL}$ after the treatments. Low-level laser therapy and kinesio taping should be kept in mind as alternative techniques for appropriate cases in the treatment of lymphedema.
\end{abstract}

Keywords: Kinesio taping, low-level laser therapy, lymphedema.

Secondary lymphedema mainly occurs by impairment or obstruction of the lymphatic system due to disease or iatrogenic procedures ${ }^{[1,2]}$ Malignancy and its treatment processes are the most common causes of secondary lymphedema in developed countries. ${ }^{[3]}$ Early and appropriate treatment options are important in reducing complications and improving prognosis. Complex decongestive therapy (CDT) is recognized as the best management technique for lymphedema, which includes compression therapy, manual lymphatic drainage (MLD), exercise, and skin care ${ }^{[2]}$ Compression therapy in reducing upper extremity lymphedema volume should be considered as the first treatment choice. ${ }^{[4]}$

Kinesio taping for edema conditions is assumed to create variations in pressure with resultant tissue deformation when applied correctly with convolutions. Decongestion of the lymphatic fluid that pooled under the skin is one of the physiological effects of kinesio taping. ${ }^{[5]}$ Kinesio taping is considered to be effective in the management of lymphedema, it became a new adjunctive treatment option in physical therapy practice, and was admitted to be useful on body parts where compression therapy or garment fitting is problematic. ${ }^{[6,7]}$

Low-level laser therapy (LLLT) has various treatment effects in rehabilitation. Low-level laser therapy (wave lengths 650-1000 nm) is a U.S. Food and Drug Administration-approved therapeutic intervention for treatment of upper extremity lymphedema and is recognized to stimulate lymphatic movement and lymphangiogenesis, soften fibrotic tissues, and improve the contractility of the lymphatic vessels that assist the lymph transport. ${ }^{[8]}$ These mechanisms enhance the movement of the fluid accumulated in the extracellular area to the lymphatic system. Although CDT is the standard care for the lymphedema treatment, we encounter various patient profiles in our clinical practice and may need to apply alternative treatment options. In this article, we report a patient with breast cancer-related lymphedema (BCRL) and arteriovenous (AV) fistula for hemodialysis in the same arm, treated

Corresponding author: Zeynep Alev Özçete, MD. Sağlık Bilimleri Üniversitesi İzmir Tepecik Eğitim ve Araştırma Hastanesi, Fizik Tedavi ve Rehabilitasyon Kliniği, 35020 Konak, İzmir, Türkiye. e-mail: zeynepozcete@gmail.com 
with LLLT and kinesio taping as compression was contraindicated to the AV fistula.

\section{CASE REPORT}

We report a 57-year-old female patient who was diagnosed as lobular carcinoma in the left breast and had bilateral total mastectomy and left axillary dissection in December 2016. She received chemotherapy with four sessions of docetaxel and cyclophosphamide and radiotherapy treatment for 25 sessions to the chest and the left axillary region. She had end stage renal disease that had been treated with three hemodialysis cycles every week for eight years and an AV fistula in the left arm because of this treatment. She had no complaints of edema or swelling on the left arm before the breast cancer surgery. A written informed consent was obtained from the patient.

The patient was admitted to our lymphedema unit with complaints of tightness and heaviness in her left arm two months following the surgery in February 2017. Upon initial evaluation, we took the circumferential measurements on the first and fifth metacarpophalangeal joints and the wrist and repeated the process for every $5 \mathrm{~cm}$ from the wrist proximally to the top of the upper limb. The limb volumes were calculated by truncated cone formula using the circumference measurements. ${ }^{[9]}$ The volume difference was $203 \mathrm{~mL}$ (right arm: $1814 \mathrm{~mL}$, left arm: $2017 \mathrm{~mL}$ ). The maximum circumferential difference was $3.6 \mathrm{~cm}$ on the sixth $5-\mathrm{cm}$ distance
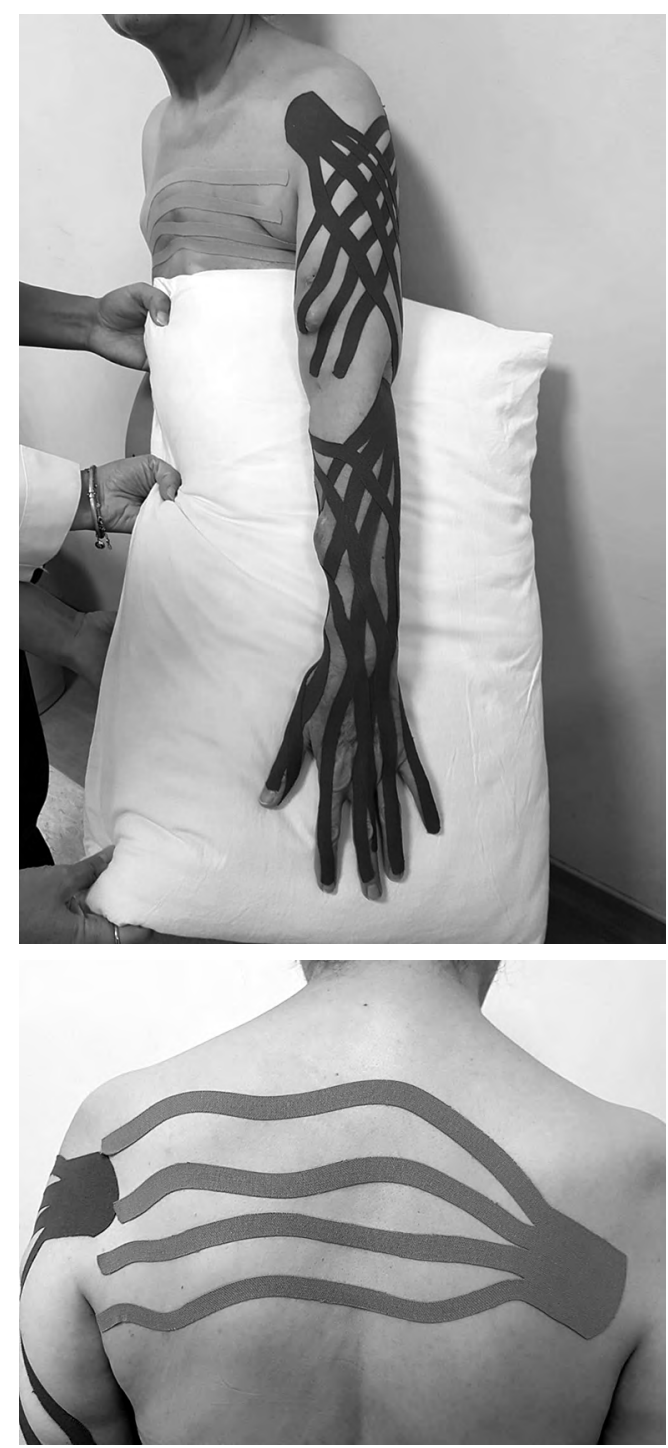

Figure 1. Kinesio tape application.
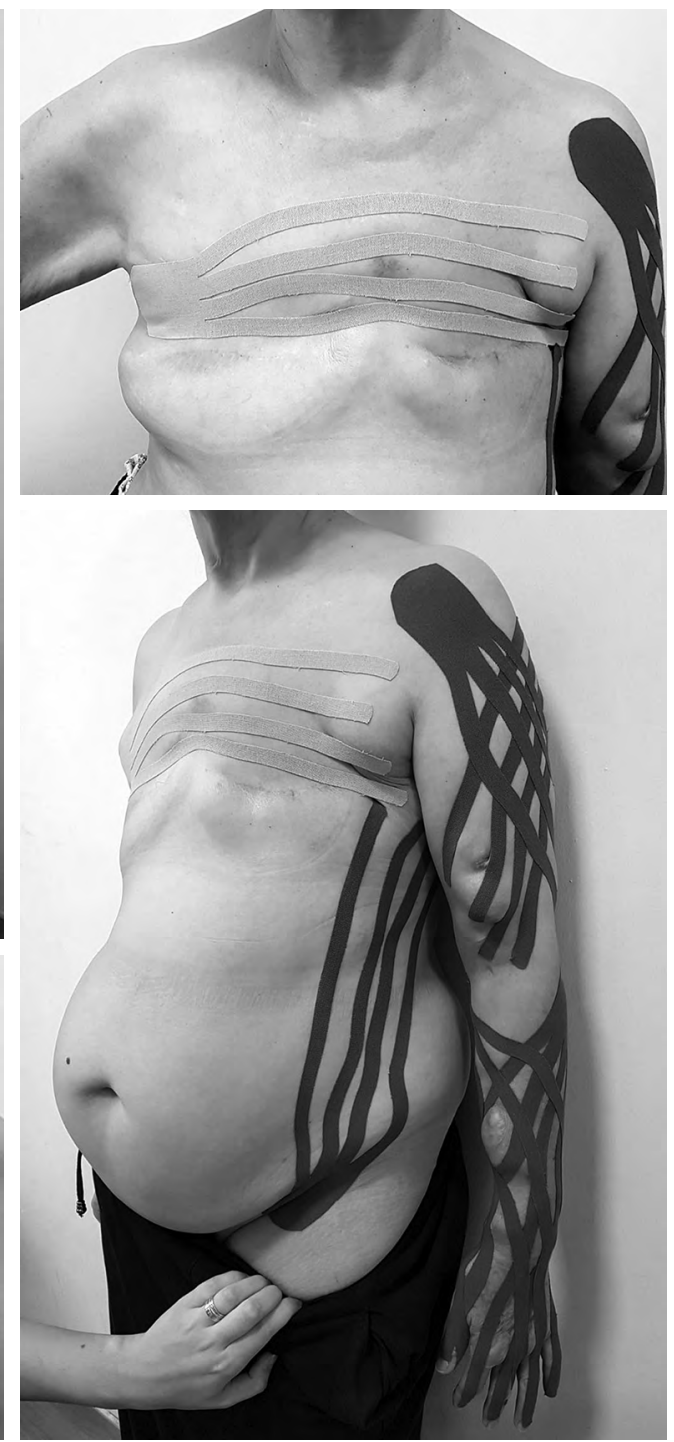
from the wrist. The Stemmer sign and pitting edema were negative. Based on the International Society of Lymphology, we recognized that the patient had stage 0 lymphedema on her left arm. The compression garment was contraindicated because of the AV fistula, thus remedial exercises including relaxation, breathing, active range of motion, and pumping exercises were given. A modified self-manual lymphatic drainage training to avoid the lymph fluid directing toward the shunt and skin care education were also performed.

In May 2017, the patient came for her follow-up visit and the volume difference of the upper limbs was $691 \mathrm{~mL}$ (right arm: $1777 \mathrm{~mL}$, left arm: $2468 \mathrm{~mL}$ ). The circumferential difference on the sixth $5 \mathrm{~cm}$ from the wrist was measured as $4.8 \mathrm{~cm}$ and the maximum circumferential difference was $5.7 \mathrm{~cm}$ on the second $5 \mathrm{~cm}$ from the wrist. The patient was recognized as stage 1 lymphedema and we decided to start LLLT and kinesio taping since compression therapy could not be performed on the arm. We tested the kinesio tape (Kinesio Tex KT-X-050, Tokyo, Japan) on an uninvolved area for any type of skin irritation and allergic reaction prior to the first application. Following 24 hours from testing, fan-shaped cut kinesio tape was applied on the left upper limb, upper anterior and posterior trunk for redirecting lymph flow from the left upper limb through the right axillary area, and on the left axillo-inguinal anastomosis for redirecting lymph flow through the left inguinal area (Figure 1). Kinesio tape was retained for three days and reapplied in the following visit. Low-level laser therapy was arranged as three therapy sessions every week for a total of 12 sessions during four weeks. Low-level laser therapy was administered at three points on the antecubital fossa and at five points on the axillary area for a total of 15 minutes $\left(5,000 \mathrm{~Hz}, 0.8 \mathrm{~J} / \mathrm{cm}^{2}\right)$ with Endolaser 422 laser device (Enraf-Nonius, Rotterdam, Netherlands). After the 12 therapy sessions, the volume difference of the upper limbs was measured as $461 \mathrm{~mL}$ (right arm: $1783 \mathrm{~mL}$, left arm: $2244 \mathrm{~mL}$ ) and the reduction of the difference was $230 \mathrm{~mL}$. We performed three extra sessions of the LLLT and kinesio taping. The patient had mild pruritus in the kineso taping area but had no wound. She was reevaluated and when the volume difference $(454 \mathrm{~mL})$ did not reveal any reduction, we ended the therapy. She was advised to perform daily exercises, self-manual lymphatic drainage, and skin care as indicated initially.
The patient came for her follow-up visit three months following the therapy in August 2017. The volume difference of the upper limbs was $454 \mathrm{~mL}$ (right arm: $1807 \mathrm{~mL}$, left arm: $2261 \mathrm{~mL}$ ) and the maximum circumferential difference was $4.6 \mathrm{~cm}$ on the sixth $5 \mathrm{~cm}$ from the wrist. The patient's last follow-up visit was in October 2018 and volume difference was $267 \mathrm{~mL}$ (right arm: $1934 \mathrm{~mL}$, left arm: $2201 \mathrm{~mL}$ ) and the maximum circumferential difference was $3.7 \mathrm{~cm}$ on the sixth $5 \mathrm{~cm}$ from the wrist.

\section{DISCUSSION}

Complex decongestive therapy program combining compression therapy (multilayer bandage or garment), manual lymphatic drainage, exercise, and skin care is considered to be the best treatment option in lymphedema management. ${ }^{[10]}$ In circumstances where compression or multilayer bandage therapy cannot be applied due to patient's poor compliance or contraindicated situations, alternative techniques can be used. In a systematic review, Kasawara et al. ${ }^{[1]}$ concluded that kinesio taping is effective in reducing the limb volume in BCRL. Similarly, the Lymphedema Framework states that kinesio taping may have a role in the management of midline edema, since compression therapy may not be well tolerated. ${ }^{[7]}$ Elasticity and lifting effect of the taping material create a massaging effect during the daily activities and exercises. The lifting of the superficial skin decreases the pressure and opens the initial lymphatics. This helps transporting of edema by leading the fluid towards a less congested lymphatic pathway and lymph node. ${ }^{[5]}$ In lymphedema management, this particular effect of kinesio taping is very important, particularly in patients with pressure sensitive skin. There is only one case study in the literature who had unilateral BCRL and AV fistula for hemodialysis in the same arm. ${ }^{[12]}$ In that case, Chou et al. ${ }^{[12]}$ arranged three MLD sessions every week for a total of 12 sessions, as well as kinesio taping and remedial exercises for the treatment. The patient's lymphedema improved from severe to moderate (volume difference reduced from $992 \mathrm{~mL}$ to $536 \mathrm{~mL}$ ). They concluded that kinesio taping could not replace the compression therapy, but it could be another option for patients in which compression therapy is contraindicated. In our patient's therapy, we used kinesio taping and also added LLLT to the treatment in order to increase efficiency. 
Wound formation by kinesio taping is a major problem, particularly in renal disease patients with uremia because pruritus, frail skin, and xerosis are common mucocutaneous disorders in those patients. Additionally, application and removal of the taping material require specific attention because the skin integrity is important to prevent any infection in lymphedema patients. Our patient did not have any complication related with kinesio taping except some pruritus in the area of application.

The LLLT is a safe and non-invasive form of phototherapy which has been encouraged and researched for the management of BCRL for over two decades. It has been used for reducing inflammation, improving lymphatic motility, preventing fibrosis, and promoting vessel regeneration. ${ }^{[13]}$ There is no standard treatment protocol for energy densities, application sites, and treatment cycles. Nevertheless, previous studies demonstrated that LLLT is a possible useful treatment for lymphedema. ${ }^{[14]}$ According to a recent systematic review, LLLT showed favorable results in reducing limb volume in comparison to sham and when no other treatment is involved, while LLLT was not more effective than any other conventional treatments. ${ }^{[15]}$ In these studies, the most commonly used energy densities were between $1.5-2 \mathrm{~J} / \mathrm{cm}^{2}$ but there were no data and dose suggestion in the literature for the use on the limb with AV fistula. Therefore, we applied $0.8 \mathrm{~J} / \mathrm{cm}^{2}$ to our patient and we suggest that this dose is effective in terms of treatment.

There is another case report that had stage 3 lymphedema due to several AV fistulas and AV graft procedures for hemodialysis. ${ }^{[16]}$ In that case, the patient had not been treated for the lymphedema and the authors did not give a treatment recommendation. The different aspect of our case was that she had no edema or swelling in her left arm with AV fistula before the breast cancer surgery. Our treatment strategy, which involved using LLLT, kinesio taping, self-manual lymphatic drainage, remedial exercises, and skin care ensured a reduction in the arm volume and this result was consistent with previous studies which demonstrated that LLLT or kinesio taping was effective in reducing edema. ${ }^{[10,11,13-15]}$

In conclusion, in cases where compression therapy is contraindicated or cannot be performed, different supplementary techniques can be used alone or in combination for the management of lymphedema. Low-level laser therapy and kinesio taping should be kept in mind as alternative techniques for appropriate cases in the treatment of lymphedema.

\section{Declaration of conflicting interests}

The authors declared no conflicts of interest with respect to the authorship and/or publication of this article.

\section{Funding}

The authors received no financial support for the research and/or authorship of this article.

\section{REFERENCES}

1. Warren AG, Brorson $H$, Borud LJ, Slavin SA. Lymphedema: a comprehensive review. Ann Plast Surg 2007;59:464-72.

2. Zuther EJ. Pathology. In: Zuther EJ, Norton S, editors. Lymphedema Management. 3rd ed. New York: Thieme; 2013. p. 46-50.

3. Morgan PA, Franks PJ, Moffatt CJ. Health-related quality of life with lymphoedema: a review of the literature. Int Wound J 2005;2:47-62.

4. McNeely ML, Magee DJ, Lees AW, Bagnall KM, Haykowsky $\mathrm{M}$, Hanson J. The addition of manual lymph drainage to compression therapy for breast cancer related lymphedema: a randomized controlled trial. Breast Cancer Res Treat 2004;86:95-106.

5. Kenzo K, Kim RS. Kinesio Taping for Lymphoedema and Chronic Swelling. Kinesio, USA: LLC; 2006.

6. Gatt M, Willis S, Leuschner S. A meta-analysis of the effectiveness and safety of kinesiology taping in the management of cancer-related lymphoedema. Eur J Cancer Care 2017;26:5.

7. Lymphoedema Framework, International Consensus. Best practice for the management of lymphoedema. MEP, London, UK; 2006

8. Ridner SH, Poage-Hooper E, Kanar C, Doersam JK, Bond SM, Dietrich MS. A pilot randomized trial evaluating lowlevel laser therapy as an alternative treatment to manual lymphatic drainage for breast cancer-related lymphedema. Oncol Nurs Forum 2013;40:383-93.

9. Casley-Smith JR, Casley-Smith J. Modern Treatment for Lymphedema. 5th ed. Adelaide: The Lymphedema Association of Australia, Inc; 1997.

10. Tsai HJ, Hung HC, Yang JL, Huang CS, Tsauo JY. Could Kinesio tape replace the bandage in decongestive lymphatic therapy for breast-cancer-related lymphedema? A pilot study. Support Care Cancer 2009;17:1353-60.

11. Kasawara KT, Mapa JMR, Ferreira V, Added MAN, Shiwa SR, Carvas N Jr, et al. Effects of Kinesio Taping on breast cancer-related lymphedema: A meta-analysis in clinical trials. Physiother Theory Pract 2018;34:337-45.

12. Chou YH, Li SH, Liao SF, Tang HW. Case report: Manual lymphatic drainage and kinesio taping in the secondary malignant breast cancer-related lymphedema in an arm with arteriovenous (A-V) fistula for hemodialysis. Am J Hosp Palliat Care 2013;30:503-6.

13. Jang DH, Song DH, Chang EJ, Jeon JY. Anti-inflammatory and lymphangiogenetic effects of low-level laser therapy on lymphedema in an experimental mouse tail model. Lasers Med Sci 2016;31:289-96. 
14. E Lima MT, E Lima JG, de Andrade MF, Bergmann A. Lowlevel laser therapy in secondary lymphedema after breast cancer: systematic review. Lasers Med Sci 2014;29:1289-95.

15. Baxter GD, Liu L, Tumilty S, Petrich S, Chapple C, Anders JJ; Laser Lymphedema Trial Team. Low level laser therapy for the management of breast cancer-related lymphedema:
A randomized controlled feasibility study. Lasers Surg Med 2018;50:924-32.

16. Maher N, Orhan S, Ahmed R. Lymphostatic Elephantiasis, Stage 3 Lymphedema in a Patient with Arteriovenous Fistulas \& Arteriovenous Grafts for Hemodialysis, a Case Report. J Cardiol Clin Res 2016;4:1072. 